\title{
TO A NONLOCAL GENERALIZATION OF THE DIRICHLET PROBLEM
}

\author{
GIVI BERIKELASHVILI
}

Received 20 August 2004; Accepted 22 September 2004

A mixed problem with a boundary Dirichlet condition and nonlocal integral condition is considered for a two-dimensional elliptic equation. The existence and uniqueness of a weak solution of this problem are proved in a weighted Sobolev space.

Copyright (c) 2006 Hindawi Publishing Corporation. All rights reserved.

\section{Introduction}

The first paper devoted to a nonlocal boundary value problem with integral conditions goes back to Cannon [1]. More general nonlocal conditions for different types of partial differential equations were considered later (see, e.g., $[2,4-6,9,11,13,14]$ ).

In the present paper, a mixed problem with a boundary Dirichlet condition and nonlocal integral condition is considered in a unit square for a second-order elliptic equation. The existence and uniqueness of a weak solution of this problem in the weighted Sobolev space $W_{2}^{1}(\Omega, \rho)$ are proved. The proof is based on the Lax-Milgram lemma. It is shown that a nonlocal problem can be regarded as a generalization of the Dirichlet boundary value problem.

\section{Statement of the problem}

Let $\Omega=\left\{\left(x_{1}, x_{2}\right): 0<x_{k}<1, k=1,2\right\}$ be a unit square with boundary $\Gamma, \Gamma_{1}=\left\{\left(0, x_{2}\right)\right.$ : $\left.0<x_{2}<1\right\}, \Gamma_{*}=\Gamma \backslash \Gamma_{1}, \Omega_{\xi}=(0, \xi) \times(0,1)$, let $\xi$ be a fixed point from $(0,1]$.

By $L_{2}(\Omega, \rho)$ we denote a weighted Lebesgue space of all real-valued functions $u(x)$ on $\Omega$ with the inner product and the norm

$$
(u, v)_{\rho}=\int_{\Omega} \rho(x) u(x) v(x) d x, \quad\|u\|_{\rho}=(u, u)_{\rho}^{1 / 2}
$$

The weighted Sobolev space $W_{2}^{1}(\Omega, \rho)$ is usually defined as a linear set of all functions $u(x) \in L_{2}(\Omega, \rho)$ whose derivatives $\partial u / \partial x_{k}, k=1,2$ (in the generalized sense), belong 
2 To a nonlocal generalization of the Dirichlet problem

to $L_{2}(\Omega, \rho)$. It is a normed linear space if equipped with the norm

$$
\|u\|_{1, \rho}=\left(\|u\|_{\rho}^{2}+|u|_{1, \rho}^{2}\right)^{1 / 2}, \quad|u|_{1, \rho}^{2}=\left\|\frac{\partial u}{\partial x_{1}}\right\|_{\rho}^{2}+\left\|\frac{\partial u}{\partial x_{2}}\right\|_{\rho}^{2} .
$$

Let us choose a weight function $\rho(x)$ as follows:

$$
\rho(x)=\left\{\begin{array}{ll}
\left(x_{1} / \xi\right)^{\varepsilon}, & x_{1} \leq \xi, \\
1, & x_{1}>\xi,
\end{array} \quad \varepsilon \in(0,1)\right.
$$

It is well known (see, e.g., [7, page 10], [10, Theorem 3.1]) that $W_{2}^{1}(\Omega, \rho)$ is a Banach space and $C^{\infty}(\bar{\Omega})$ is dense in $W_{2}^{1}(\Omega, \rho)$ and in $L_{2}(\Omega, \rho)$. As an immediate consequence, we can define the space $W_{2}^{1}(\Omega, \rho)$ as a closure of $C^{\infty}(\bar{\Omega})$ with respect to the norm $\|\cdot\|_{1, \rho}$ and these both definitions are equivalent.

Define the subspace of the space $W_{2}^{1}(\Omega, \rho)$ which can be obtained by closing the set $C^{*}(\bar{\Omega})=\left\{u \in C^{\infty}(\bar{\Omega}): \operatorname{supp} u \cap \Gamma_{*}=\varnothing, l(u)=0,0<x_{2}<1\right\}$ with the norm $\|\cdot\|_{1, \rho}$. Denote it by $W_{2}^{1}(\Omega, \rho)$.

For $f \in L_{\infty}(\Omega)$, denote $\|f\|_{\infty}=\operatorname{vraimax}_{x \in \Omega}|f(x)|$.

We say that the function $b$ has the property $(\mathrm{P})$ if $b \in L_{\infty}(\Omega)$ and $0 \leq x_{1}^{\varepsilon} \partial\left(x_{1}^{1-\varepsilon} b\right) / \partial x_{1} \in$ $L_{\infty}\left(\Omega_{\xi}\right)$.

Consider the nonlocal boundary value problem

$$
\mathscr{L} u=f(x), \quad x \in \Omega, \quad u(x)=0, \quad x \in \Gamma_{*}, \quad l(u)=0, \quad 0<x_{2}<1,
$$

where

$$
\mathscr{L} u=\sum_{i, j=1}^{2} \frac{\partial}{\partial x_{i}}\left(a_{i j} \frac{\partial u}{\partial x_{j}}\right)-a_{0} u, \quad l(u)=\int_{0}^{\xi} \beta(x) u(x) d x_{1},
$$

$\beta(x)=\varepsilon x_{1}^{\varepsilon-1} / \xi^{\varepsilon}$ if $x_{1} \leq \xi, \rho(x)=0$ if $x_{1}>\xi$.

Let the right-hand side $f(x)$ in $(2.4)$ be a linear continuous functional on ${ }_{W}^{*}(\Omega, \rho)$ which can be represented as

$$
f=f_{0}+\frac{\partial f_{1}}{\partial x_{1}}+\frac{\partial f_{2}}{\partial x_{2}}, \quad f_{k}(x) \in L_{2}(\Omega, \rho), k=0,1,2 .
$$

We assume that the coefficients $a_{i j}$ and $a_{0}$ satisfy the following conditions:

$$
\begin{gathered}
a_{1 j}=a_{1 j}(x) \in L_{\infty}(\Omega) \quad(j=1,2) \quad a_{21}=\text { const; } \\
a_{22} \text { and } a_{0} \text { have property }(\mathrm{P}), \\
a_{0} \geq 0, \quad \sum_{i, j=1}^{2} a_{i j} t_{i} t_{j} \geq v\left(t_{1}^{2}+t_{2}^{2}\right) \quad \text { a.e. in } \Omega, \nu=\text { const }>0 .
\end{gathered}
$$


We say that the function $u \in W_{2}^{*}(\Omega, \rho)$ is a weak solution of problem (2.4)-(2.7) if the relation

$$
a(u, v)=\langle f, v\rangle, \quad \forall v \in W_{2}^{*}(\Omega, \rho)
$$

holds, where

$$
\begin{gathered}
a(u, v)=\left(a_{11} \frac{\partial u}{\partial x_{1}}, \frac{\partial v}{\partial x_{1}}\right)_{\rho}+\left(\left(a_{12}+a_{21}\right) \frac{\partial u}{\partial x_{2}}, \frac{\partial v}{\partial x_{1}}\right)_{\rho}+\left(a_{22} \frac{\partial u}{\partial x_{2}}, K \frac{\partial v}{\partial x_{2}}\right)_{\rho}+\left(a_{0} u, K v\right)_{\rho} \\
\langle f, v\rangle=\left(f_{0}, K v\right)_{\rho}-\left(f_{1}, \frac{\partial v}{\partial x_{1}}\right)_{\rho}-\left(f_{2}, K \frac{\partial v}{\partial x_{2}}\right)_{\rho} \\
K v(x)=v(x)-\frac{1}{\rho\left(x_{1}\right)} \int_{0}^{x_{1}} \beta(t) v\left(t, x_{2}\right) d t
\end{gathered}
$$

Equality (2.8) is formally obtained from $(\mathscr{L} u-f, K v)_{\rho}=0$ by integration by parts.

\section{Solvability of a nonlocal problem}

To prove the existence of a unique solution of problem (2.8) (a weak solution of the problem (2.4)-(2.7)), we will apply Lax-Milgram lemma [3]. First we will prove some auxiliary statements.

Lemma 3.1. Let $u \in{ }^{*} W_{2}^{1}(\Omega, \rho)$. Then

$$
|u|_{1, \rho} \leq\|u\|_{1, \rho} \leq c_{2}|u|_{1, \rho}, \quad c_{2}=\sqrt{5}
$$

Proof. The first inequality of the lemma is obvious. Integrating by parts, we obtain

$$
\begin{aligned}
\int_{\Omega} \rho(x) u^{2}(x) d x= & -\frac{\varepsilon \xi}{\varepsilon+1} \int_{0}^{1} u^{2}\left(\xi, x_{2}\right) d x_{2}-2 \int_{\xi}^{1} \int_{0}^{1} x_{1} u(x) \frac{\partial u}{\partial x_{1}} d x \\
& -\frac{2}{\varepsilon+1} \int_{0}^{\xi} \int_{0}^{1} x_{1} \rho(x) u(x) \frac{\partial u}{\partial x_{1}} d x \leq 2 \int_{\Omega} \rho(x)\left|u \frac{\partial u}{\partial x_{1}}\right| d x
\end{aligned}
$$

Therefore

$$
\|u\|_{\rho} \leq 2\left\|\frac{\partial u}{\partial x_{1}}\right\|_{\rho}
$$

which proves the lemma.

Lemma 3.2. Let $u, v \in L_{2}(\Omega, \rho)$ and let $v$ satisfy the condition $l(v)=0$. Then

$$
\|v\|_{\rho} \leq\|K v\|_{\rho} \leq c_{1}\|v\|_{\rho}
$$


4 To a nonlocal generalization of the Dirichlet problem

further, if $b$ belongs to $L_{\infty}(\Omega)$, then

$$
\left|(b u, K v)_{\rho}\right| \leq c_{1}\|b\|_{\infty}\|u\|_{\rho}\|v\|_{\rho},
$$

where $c_{1}=(1+\varepsilon) /(1-\varepsilon)$.

Proof. Denote

$$
J(v)=\int_{\Omega_{\xi}} \rho^{-1}(x)\left(\int_{0}^{x_{1}} \beta(t) v\left(t, x_{2}\right) d t\right)^{2} d x .
$$

It is not difficult to verify that

$$
J(v)=-\frac{2 \varepsilon}{1-\varepsilon} \int_{\Omega_{\xi}} v(x) \int_{0}^{x_{1}} \beta(t) v\left(t, x_{2}\right) d t d x \leq \frac{2 \varepsilon}{1-\varepsilon}\|v\|_{\rho} J(v) .
$$

Thus

$$
(J(v))^{1 / 2} \leq 2 \varepsilon(1-\varepsilon)^{-1}\|v\|_{\rho}
$$

Since

$$
\int_{0}^{x_{1}} \beta(t) v\left(t, x_{2}\right) d t=0, \quad x_{1} \geq \xi
$$

from (2.11) we get

$$
\|K v\|_{\rho}^{2}=\|v\|_{\rho}^{2}+\left(\frac{1}{\varepsilon}\right) J(v)
$$

which using (3.8) yields (3.4).

Further, we can write

$$
(b u, K v)_{\rho}=(b u, v)_{\rho}-\int_{\Omega_{\xi}} b u \int_{0}^{x_{1}} \beta(t) v\left(t, x_{2}\right) d t d x,
$$

and by virtue of the Cauchy inequality we have

$$
\left|(b u, K v)_{\rho}\right| \leq\|b\|_{\infty}\|u\|_{\rho}\left(\|v\|_{\rho}+(J(v))^{1 / 2}\right) .
$$

This together with (3.8) completes the proof of (3.5).

Lemma 3.3. Let $v \in L_{2}(\Omega, \rho)$ and $l(v)=0$. If $b$ has property $(P)$, then

$$
(b v, K v)_{\rho} \geq(b v, v)_{\rho}
$$

The proof follows from the easily verifiable identity

$$
(b v, K v)_{\rho}=(b v, v)_{\rho}-\int_{\Omega_{\xi}} b v \int_{0}^{x_{1}} \beta(t) v\left(t, x_{2}\right) d t d x=(b v, v)_{\rho}+\frac{1}{2 \varepsilon} \tilde{J},
$$


where

$$
\tilde{J}=\int_{\Omega_{\xi}} x_{1}^{\varepsilon} \frac{\partial\left(x_{1}^{1-\varepsilon} b\right)}{\partial x_{1}} \rho^{-1}\left(\int_{0}^{x_{1}} \beta(t) v\left(t, x_{2}\right) d t\right)^{2} d x<\infty .
$$

By applying Lemmas 3.1, 3.2, 3.3, and conditions (2.7), from (2.9) we obtain the continuity

$$
|a(u, v)| \leq c_{3}\|u\|_{1, \rho}\|v\|_{1, \rho}, \quad c_{3}>0, \forall u, v \in W_{2}^{*}(\Omega, \rho)
$$

and the $W_{2}^{1}$-ellipticity

$$
a(u, u) \geq c_{4}\|u\|_{1, \rho}^{2}, \quad c_{4}>0, \forall u \in \stackrel{*}{*} W_{2}^{1}(\Omega, \rho)
$$

of the bilinear form $a(u, v)$.

Analogously, from (2.10) follows the continuity of the linear form $\langle f, v\rangle$ :

$$
|\langle f, v\rangle| \leq c_{5}\|v\|_{1, \rho}, \quad c_{5}>0, \forall v \in W_{2}^{*}(\Omega, \rho) .
$$

Thus, all conditions of the Lax-Milgram lemma are fulfilled. Therefore the following theorem is true.

Theorem 3.4. The problem (2.4)-(2.7) has a unique weak solution from ${ }^{*} W_{2}^{1}(\Omega, \rho)$.

Remark 3.5. If we notice that

$$
u\left(\xi, x_{2}\right)-l(u)=\int_{0}^{\xi} \rho\left(x_{1}\right) \frac{\partial u(x)}{\partial x_{1}} d x_{1},
$$

then, applying the Cauchy inequality, we get

$$
\left|\int_{0}^{1}\right| u\left(\xi, x_{2}\right)-\left.l(u)\right|^{2} d x_{2} \mid \leq c_{6}\|u\|_{1, \rho}^{2}, \quad c_{6}=\frac{\xi}{\varepsilon+1} .
$$

Consequently,

$$
\lim _{\xi \rightarrow 0} l(u)=u\left(0, x_{2}\right)
$$

Thus, passing to the limit as $\xi \rightarrow 0$, the nonlocal condition $l(u)=0$ transforms to $u\left(0, x_{2}\right)=0$, while Theorem 3.4 transforms to the well-known theorem on the existence and uniqueness of a solution of the Dirichlet problem. In this sense, the nonlocal problem (2.4)-(2.7) can be regarded as a generalization of the Dirichlet boundary value problem.

Remark 3.6. By definition (2.9), for all $u \in D(\mathscr{L})$ we have $a(u, u)=(\mathscr{L} u, K u)_{\rho}$. Hence, using (3.4) it follows from (3.17) that

$$
(\mathscr{L} u, K u)_{\rho} \geq c\|u\|_{\rho}^{2}, \quad(\mathscr{L} u, K u)_{\rho} \geq c\|K u\|_{\rho}^{2} .
$$

Thus $\mathscr{L}$ is a $K$-positive definite operator $[8,12]$. 
6 To a nonlocal generalization of the Dirichlet problem

\section{References}

[1] J. R. Cannon, The solution of the heat equation subject to the specification of energy, Quarterly of Applied Mathematics 21 (1963), no. 2, 155-160.

[2] M. Chipot and B. Lovat, Some remarks on nonlocal elliptic and parabolic problems, Nonlinear Analysis 30 (1997), no. 7, 4619-4627.

[3] P. G. Ciarlet, The Finite Element Method for Elliptic Problems, Studies in Mathematics and Its Applications, vol. 4, North-Holland, Amsterdam, 1978.

[4] H. De Schepper and M. Slodička, Recovery of the boundary data for a linear second order elliptic problem with a nonlocal boundary condition, ANZIAM Journal 42 (2000), Part C, C518-C535.

[5] D. Gordeziani and G. Avalishvili, Investigation of the nonlocal initial boundary value problems for some hyperbolic equations, Hiroshima Mathematical Journal 31 (2001), no. 3, 345-366.

[6] A. K. Gushchin and V. P. Mikhailov, On the solvability of nonlocal problems for a second-order elliptic equation, Matematicheskiǔ Sbornik 185 (1994), no. 1, 121-160 (Russian), translated in Russian Academy of Sciences Sbornik Mathematics 81 (1995), no. 1, 101-136.

[7] A. Kufner and A.-M. Sändig, Some Applications of Weighted Sobolev Spaces, Teubner-Texte zur Mathematik, vol. 100, BSB B. G. Teubner, Leipzig, 1987.

[8] A. E. Martynyuk, Some new applications of methods of Galerkin type, Matematicheskii Sbornik 49 (91) (1959), 85-108 (Russian).

[9] S. Mesloub, A. Bouziani, and N. Kechkar, A strong solution of an evolution problem with integral conditions, Georgian Mathematical Journal 9 (2002), no. 1, 149-159.

[10] A. Nekvinda and L. Pick, A note on the Dirichlet problem for the elliptic linear operator in Sobolev spaces with weight $d_{M}^{\epsilon}$, Commentationes Mathematicae Universitatis Carolinae 29 (1988), no. 1, 63-71.

[11] B. P. Paneyakh, Some nonlocal boundary value problems for linear differential operators, Matematicheskie Zametki 35 (1984), no. 3, 425-434 (Russian).

[12] W. V. Petryshyn, On a class of $K$ - p.d. and non $K$-p.d. operators and operator equations, Journal of Mathematical Analysis and Applications 10 (1965), no. 1, 1-24.

[13] M. P. Sapagovas, A difference scheme for two-dimensional elliptic problems with an integral condition, Litovskii Matematicheskii Sbornik 23 (1983), no. 3, 155-159 (Russian).

[14] A. L. Skubachevskii and G. M. Steblov, On the spectrum of differential operators with a domain that is not dense in $L_{2}(0,1)$, Doklady Akademii Nauk SSSR 321 (1991), no. 6, 1158-1163 (Russian), translated in Soviet Mathematics Doklady 44 (1992), no. 3, 870-875.

Givi Berikelashvili: A. Razmadze Mathematical Institute, Georgian Academy of Sciences, 1 M. Aleksidze Street, Tbilisi 0193, Georgia, Caucasus

E-mail address: bergi@rmi.acnet.ge 